\title{
Efficacy of montelukast in the management of COVID-19: double blind randomized placebo controlled trial
}

\author{
Vijay Kumar ${ }^{1 *}$, Avinash A. Ganapule1, Sushmita Supriya ${ }^{1}$, Divendu Bhushan ${ }^{1}$, Pallavi Lohani², \\ Sanjay Pandey², B. Hilbert Sahoo', Anjani Kumar', Shruti Singh ${ }^{3}$, Ramesh Kumar ${ }^{4}$
}

${ }^{1}$ Department of General Medicine, ${ }^{2}$ Department of CFM, ${ }^{3}$ Department of Pharmacology, ${ }^{4}$ Department of Gastroenterology, All India Institute of Medical Sciences, Patna, Bihar, India

Received: 29 September 2021

Revised: 29 October 2021

Accepted: 30 October 2021

\section{*Correspondence:}

Dr. Vijay Kumar,

Email: vk1994@gmail.com

Copyright: (C) the author(s), publisher and licensee Medip Academy. This is an open-access article distributed under the terms of the Creative Commons Attribution Non-Commercial License, which permits unrestricted non-commercial use, distribution, and reproduction in any medium, provided the original work is properly cited.

\section{ABSTRACT}

Background: Objective of the study was to determine the efficacy of montelukast in reducing the severity of COVID19 symptoms using a double blinded randomized controlled trial.

Methods: Parallel, double-blinded randomized controlled trial (RCT) with placebo as comparison to montelukast. All patients above the age of 14 years both males and females, admitted with a diagnosis of mild or moderate COVID-19 (on the basis of a positive reverse transcriptase polymerase chain reaction (RT-PCR) report) at our facility during the study period from 01 September 2020-31 January 2021) and excluding those having adverse reaction to montelukast or those not willing to participate, and pregnant and lactating females. Patients in the intervention arm were given tablet montelukast $10 \mathrm{mg}$ OD HS from the day of admission for 10 days. The patients in the placebo group were given an identical appearing placebo at bedtime for 10 days from the day of admission. The rest of the treatment was given as per the standard operating procedure (SOP) of the institute with minor adjustments as per the treating team's judgement. Primary outcome was progression of the disease to severe grade and secondary outcomes were discharge on or before day 10 from admission, admission to ICU, need for mechanical ventilation and in-hospital mortality.

Results: A total of 94 patients were enrolled for the study. 90 patients, 45 in each arm were included in the final analysis. The baseline characteristics of the two arms including age, sex, comorbidities, severity at admission and treatment given apart from montelukast or placebo, were comparable with respect to these variables. This study did not find any improvement in primary outcome of progression to severe disease and secondary outcomes of intensive care unit (ICU) admission, mortality or need of mechanical ventilation, discharge on or by day 10 with the use of montelukast as compared to placebo in mild to moderate cases of COVID-19.

Conclusions: There was no difference in primary or secondary outcomes with the use of Montelukast compared to placebo.

Keywords: COVID-19, Montelukast, Randomized controlled trial

\section{INTRODUCTION}

The severe acute respiratory syndrome coronavirus-2 (SARS-CoV-2) infection first reported from Wuhan in China in November 2019 has grown into a pandemic affecting almost all regions of the world. ${ }^{1}$
The pandemic, emerged with the new coronavirus 2019 (COVID-19), has not yet been brought under control, despite serious measures taken all over the world and efforts to control and treat the disease. Up till now, a specific treatment for COVID-19 infection is not available. 
Though several treatments have been tried, none has been convincingly found to be effective except low dose steroids in moderate-severe cases. ${ }^{2}$

Drugs such as remdesivir and tocilizumab have been found to be useful in specific patient populations. The anti-viral drug Remdesivir is especially useful in shortening time to recovery, but the interim results of the World Health Organization (WHO) solidarity trial have questioned its efficacy.,4 Tocilizumab has been found to reduce progression to mechanical ventilation in specific subgroups such as those with recent shift to intensive care unit (ICU) and raised inflammatory markers. ${ }^{5}$ Also, preliminary results of the recovery trial have suggested a mortality benefit. ${ }^{6}$

As is evident in the use of the above drugs, some of these have been used earlier for other diseases and conditions. Yet they have been repurposed for use in COVID-19. This is often the situation in pandemics where existing drugs have to often be repurposed based on mechanisms which might explain their possible role in the control of the disease. ${ }^{7}$ As pandemics surge rapidly, this is often a better option than to explore newer drugs specific for the new disease as the pharmacological profile of the repurposed drugs is well known.

Some such approaches to treatment should be considered in cases of COVID-19. These may include ivermectin, an anti-helminthic drug, azithromycin, an antibiotic, montelukast, a cysteinyl leukotriene (cysLT) receptor antagonist and a few others. Even combinations of these drugs are being studied. ${ }^{8-11}$

The clinical features of COVID-19 can range from asymptomatic cases to acute respiratory distress syndrome (ARDS) and multiorgan dysfunction. The most important cause of COVID-19 related deaths is respiratory failure, which is progressive and unresponsive to treatment. ${ }^{12}$ ARDS, which frequently occurs in these patients, is an acute inflammatory lung injury, a clinical condition that is not well understood due to its complex pathogenesis, and is a result of widespread alveolar injury caused by intense inflammation. IL-6, IL-8, the tumour necrosis factor (TNF) alpha and IL-1 produced in the early phase and other proinflammatory cytokines that occur in the later stages of the disease, inducing leukocyte migration to the region. ${ }^{13}$ Then, leukocytes accumulating in the lungs are activated and secrete reactive oxygen species and proteases that damage capillary endothelium and alveolar epithelium.

Montelukast is a potent cysteinyl leukotriene (cysLT) receptor antagonist with anti-inflammatory effects and has been proven to significantly suppress oxidative stress. Also, CysLTs can affect cytokine production. ${ }^{14}$ Montelukast therapy has been shown in recent studies to reduce TNF- $\alpha$, IL- 6 and IL-1b levels amongst other cytokines. $^{15}$
Further, montelukast acts via angiotensin converting enzyme (ACE) receptors to inhibit bradykinin induced hypersensitivity. ${ }^{16}$ Because COVID-19 virus enters into the cell through ACE receptors and causes mortality due to excessive inflammatory processes, it is thought that montelukast may have a limiting effect on the progression of the disease in COVID-19 infection. Also, as it is a costeffective drug with minimal side effects, it can be a very good adjuvant therapy in COVID-19.

A preliminary observational study in elderly asthmatics on montelukast therapy showed decreased risk of acquiring COVID-19 and decrease in hospitalization due to it as compared to those not on montelukast. ${ }^{17}$ This, along with the multiple proposed mechanisms of montelukast in COVID-19 led to further studies. ${ }^{18}$ A retrospective study showed good clinical outcome after use of montelukast based on the clinical ordinal scale. ${ }^{19}$ Multiple other prospective studies and trials are ongoing..$^{20,21}$

In the current study, we are trying to assess the effect of montelukast on progression of the disease as well as its effect on shortening hospital stay.

\section{METHODS}

The study was aimed at assessing the efficacy of montelukast in preventing the progression of disease in mild-moderate cases of COVID-19.

\section{Study type}

The type of study was double blind randomized placebo controlled trial.

\section{Study place}

The study was conducted at the All India Institute of Medical Sciences (AIIMS), Patna, Bihar, India.

\section{Study period}

The period of the study was from 01 September 2020 to 31 December 2020.

\section{Ethical clearance}

Ethical clearance was obtained from the institutional ethics committee, AIIMS, Patna. The trial was registered with the clinical trials registry - India (registration numberCTRI/2020/11/029232).

\section{Sample size}

Assuming improvement of $30 \%$ in 10 day recovery in patients receiving drug $\mathrm{A}(50 \%)$ compared to drug $\mathrm{B}$ $(20 \%)$ with $5 \%$ absolute precision and $80 \%$ power, the total sample size was calculated to be 90 using Open epi software with equal allotment in both the arms. 


\section{Inclusion criteria}

All patients above the age of 14 years both males and females, admitted with a diagnosis of mild or moderate COVID-19 (on the basis of a positive reverse transcriptase-polymerase chain reaction (RT-PCR) report) at AIIMS/Patna during the study period (01 September 2020-31 December 2020) were included in the study provided they do not meet any of the exclusion criteria .

\section{Exclusion criteria}

Patients with a known allergy to or adverse drug reaction with montelukast, unable to provide consent or unwilling to participate in the study, patients included in any other ongoing trial and pregnant and lactating females were excluded from the study.

\section{Definitions of mild, moderate and severe COVID-1922}

\section{Mild}

No evidence of breathlessness or hypoxia (normal saturation).

\section{Moderate}

Breathlessness and/or hypoxia (saturation $90-94 \%$ on room air), respiratory rate of 24 or more and no features of severe disease.

\section{Severe}

Any of the following - severe respiratory distress, oxygen saturation $<90 \%$ on room air, respiratory rate $>30$, shock or evidence of a life threatening organ dysfunction

The patients, after obtaining written informed consent, were randomly assigned to either the intervention arm or the placebo arm (using block randomisation with variable block sizes of 4, 6, 8 generated by sealed envelope software). All the parameters were noted for all patients at the time of admission such as age, sex, any pre-existing comorbidities, presenting symptoms of the patients, other medications prescribed including steroids, heparin, remdesivir, convalescent plasma therapy, tocilizumab, antibiotics and vitals like heart rate, blood pressure, temperature, respiratory rate, oxygen saturation, mental status (AVPU) and qSOFA score (on admission and at least once every day).

\section{Study design}

The patients in the intervention arm were given one tablet of $10 \mathrm{mg}$ of montelukast once daily at bed time for 10 days from the time of admission. The patients in the placebo arm received one similar looking placebo tablet with identical packaging. Neither the patient nor the treating team or the researchers were aware whether an individual patient has received montelukast or placebo. Only the pharmacist dispensing the tablets was aware of the same until data analysis. Either principal investigator or coprincipal investigator provided the drug or placebo in sealed envelope to the patients. Apart from this, standard treatment was provided to all patients in both the arms, according to their clinical needs and as per the ICMR protocol and SOP of the institute. ${ }^{22}$ Patients were discharged as per the guidelines prescribed in SOP of the institute.

The discharge criteria were: ten days from symptom onset, afebrile for three days and maintaining saturation above $94 \%$ for four consecutive days without supplemental oxygen. These were applied in conjunction with the decisions made by the treating team.

The following outcomes were measured: primary outcome was progression of the disease to severe grade while secondary outcomes were admission to ICU, need for mechanical ventilation, in-hospital mortality and discharge on or before day 10 from admission.

\section{Statistical analysis}

Statistical analysis was done using IBM statistical package for the social sciences (SPSS) (Chicago, USA) software, version 22. All descriptive data were expressed as mean (SD) and frequency (percentage). Chi-square test and Fischer's exact test were performed to assess difference in the primary and secondary outcome measures between the two groups. A $\mathrm{p}$ value $<0.05$ was considered to be statistically significant.

\section{RESULTS}

A total of 94 patients were enrolled for the study, 47 of whom were randomized to the intervention group and 47 to the placebo group. One patient in the intervention group dropped out after 2 days due to safety concerns. One patient in the intervention arm and two in the placebo arm were excluded as they were given a combination containing montelukast by the treating team (Figure 1). Thus, 45 patients in each arm were included in the final analysis. Table 1 shows the baseline characteristics of the two arms including age, sex, comorbidities, severity at admission and treatment given apart from montelukast or its placebo. Both groups were comparable with respect to these variables. Table 2 shows the outcomes of the study. $22.22 \%$ patients in the intervention group as compared to $17.78 \%$ in the placebo group progressed to severe disease, although all of them eventually recovered. The difference was statistically not significant. 3 persons in the placebo group required ICU admission as compared to 1 in the intervention group. However, it was not statistically significant. There were no deaths or need for mechanical ventilation in either group although one person in the placebo group needed non-invasive ventilation. Further, $53.33 \%$ patients in both the intervention group and placebo group were discharged on or before day 10. All patients from both groups were successfully discharged. 
Table 1: Comparison of baseline characteristics of montelukast and non-montelukast group.

\begin{tabular}{|c|c|c|c|}
\hline \multirow{2}{*}{ Characteristics } & \multirow{2}{*}{$\begin{array}{l}\text { Montelukast group }[\mathrm{N}=45] \\
\text { n }(\%)\end{array}$} & \multirow{2}{*}{$\begin{array}{l}\text { Non- montelukast group }[\mathrm{N}=45] \\
\text { n (\%) }\end{array}$} & \multirow[b]{2}{*}{$P$ value } \\
\hline & & & \\
\hline Mean age & 54.3 & 52.2 & 0.504 \\
\hline Male & 68.9 & 71.1 & 0.818 \\
\hline Female & 31.1 & 28.9 & 0.818 \\
\hline Mean days from symptoms onset & 5.8 & 7 & 0.172 \\
\hline Fever & 73.3 & 66.7 & 0.49 \\
\hline Shortness of breath & 35.6 & 35.6 & 1 \\
\hline Cough & 57.8 & 46.7 & 0.291 \\
\hline Generalised weakness & 2.2 & 6.7 & 0.616 \\
\hline GI symptoms & 6.7 & 13.3 & 0.485 \\
\hline Hypertension & 42.2 & 42.2 & 1 \\
\hline Type II DM & 46.7 & 40 & 0.523 \\
\hline IHD & 13.3 & 6.7 & 0.485 \\
\hline Heart failure & 2.2 & 0 & 1 \\
\hline CKD & 4.4 & 2.2 & 1 \\
\hline COPD & 4.4 & 0 & 0.494 \\
\hline Asthma & 13.3 & 6.7 & 0.485 \\
\hline Hypothyroidism & 13.3 & 11.1 & 0.748 \\
\hline \multicolumn{4}{|l|}{ Severity at the time of admission } \\
\hline Mild & 68.9 & 82.2 & 0.141 \\
\hline Moderate & 31.1 & 17.8 & 0.141 \\
\hline History of smoking & 24.4 & 8.9 & 0.048 \\
\hline \multicolumn{4}{|l|}{ Treatment given } \\
\hline Heparin & 97.8 & 86.7 & 0.049 \\
\hline HCQS & 20 & 28.9 & 0.327 \\
\hline Corticosteroid & 88.9 & 91.1 & 1 \\
\hline Azithromycin & 84.4 & 77.8 & 0.419 \\
\hline Remdesevir & 15.6 & 11.1 & 0.535 \\
\hline
\end{tabular}

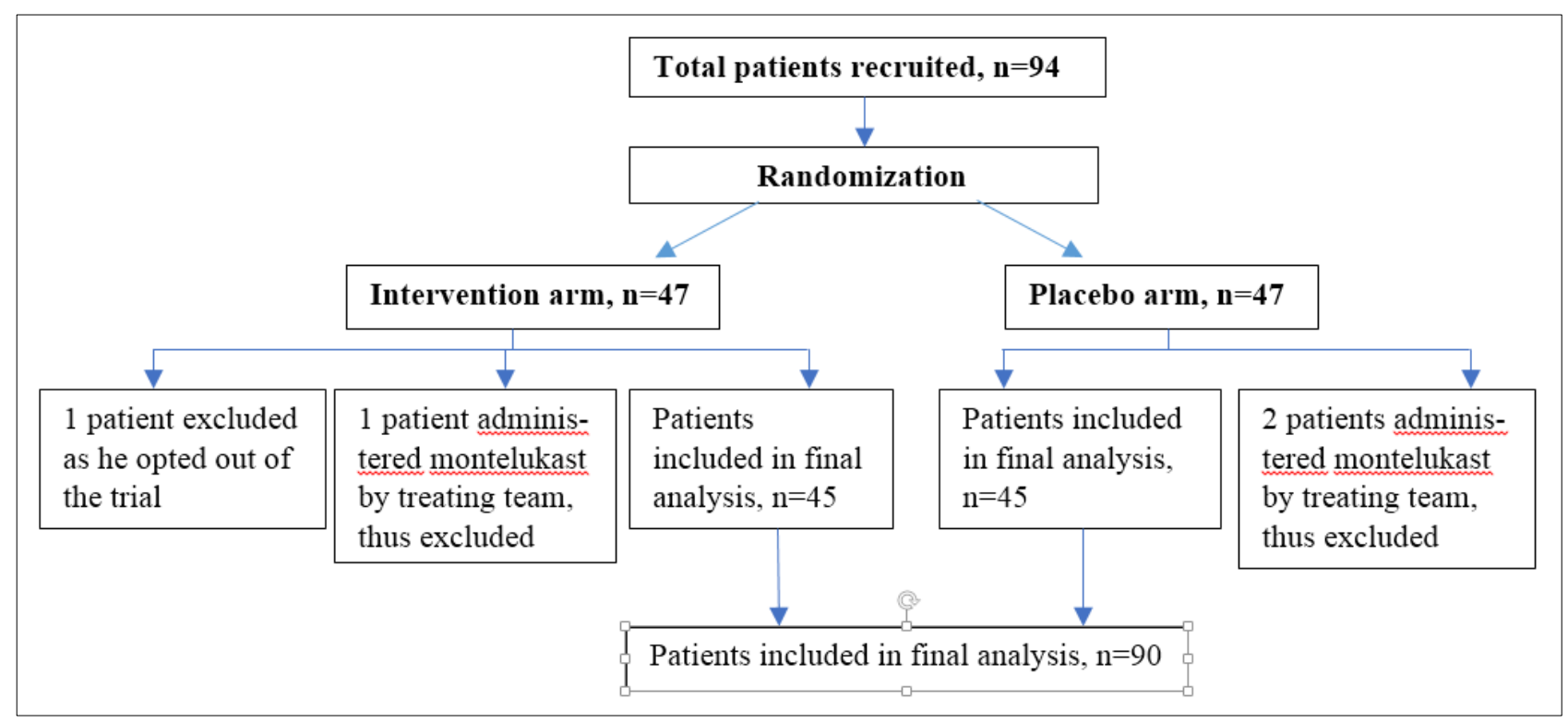

Figure 1: Intervention arm and placebo arm. 
Table 2: Distribution of study subjects according to primary and secondary outcomes.

\begin{tabular}{|c|c|c|c|}
\hline Outcome variable & $\begin{array}{l}\text { Montelukast group } \\
(\mathrm{N}=45)(\%)\end{array}$ & $\begin{array}{l}\text { Placebo group } \\
(\mathrm{N}=45)(\%)\end{array}$ & P value \\
\hline \multicolumn{4}{|l|}{ Primary outcome } \\
\hline Progression to severe disease & 22.22 & 17.78 & 0.598 \\
\hline \multicolumn{4}{|l|}{ Secondary outcomes } \\
\hline ICU admission & 2.22 & 6.67 & 0.616 \\
\hline Mortality & 0 & 0 & 1 \\
\hline Intubation & 0 & 0 & 1 \\
\hline Discharge by day 10 & 53.33 & 53.33 & 1 \\
\hline
\end{tabular}

\section{DISCUSSION}

This study did not find any improvement in primary outcome of progression to severe disease with the use of montelukast as compared to placebo in mild to moderate cases of COVID-19. Also, the secondary outcomes of discharge on or by day 10 , ICU admission, mortality or need of mechanical ventilation were not improved by use of montelukast either. In a preliminary study by Bozek et al patients of asthma taking montelukast along with other treatment had lesser risk of acquiring COVID-19 infection as compared to those not on montelukast. ${ }^{17}$ Further, there was also a reduced risk of hospitalization among those who had acquired the infection.

In a previous retrospective study by Khan et al patients treated with montelukast in addition to other routine treatment had lesser risk of clinical deterioration compared to those not treated with montelukast. ${ }^{19}$ But there was no significant reduction in laboratory values of inflammatory markers or reduction in hospital stay as compared to those not treated with montelukast. Further, a comparative effectiveness study by Lima-Morales et al evaluated the efficacy of a multi-drug regimen consisting of montelukast, ivermectin, azithromycin and aspirin in patients of COVID-19. ${ }^{11}$ This study found a better chance of discharge from hospitalization within 14 days and a reduced risk of hospitalization and death in ambulatory patients treated with the above regimen versus a comparison group although a difference in baseline characteristics of the two groups might have affected the results as well.

In the current study, only one patient in the montelukast group needed ICU admission as compared to three in the comparison group but it did not reach statistical significance, mainly due to small sample size. Montelukast did not shorten hospital stay as also observed in the study by Khan et al. ${ }^{19}$ The primary outcome assessed was that montelukast did not reduce the risk of progression to severe disease as opposed to some previous retrospective studies. We also observed that $31.1 \%$ of patients in the montelukast group had moderate disease at admission as compared to $17.8 \%$ in the placebo group, which might have affected the results. Most of the other treatment given was similar in both groups (Table 1), thus reducing the possibility of their use affecting the results. Also, during the course of the trial, no patient complained of any particular side effect after intake of drug or placebo, which is in agreement with the good side effect profile of montelukast. Also, there were certain discrepancies in the baseline treatment like use of corticosteroids in some of the mild cases as well, but these were stopped after discussion with consultant on subsequent rounds. Few patients did not receive azithromycin and some were given enoxaparin in mild cases which went against the SOP due to either the treating team's decision or oversight or patient refusal. The trial was subject to certain limitations which might have affected the results. As a majority of the cases were mild and very few progressed to a state where ICU admission was needed, we could not achieve statistically significant samples to assess this particular outcome. Also, we could not assess the efficacy of the drug in severe cases. Further, as we did not correlate use of the drug with levels of inflammatory markers, a quantitative and objective assessment of the use of the drug in suppressing the cytokine storm could not be assessed. Larger studies with repeat inflammatory markers sent prior to and after use of montelukast might overcome these limitations.

\section{Limitations}

The study was double-blinded RCT, thus reducing chance of bias. Most of the outcomes were objective, not subjective. Both outcomes relating to progression of disease severity and in terms of hastening recovery were measured. Severe cases were not included in the study, hence results might not be applicable to those patients. As there was no mortality, some of the primary outcome measures could not be assessed. As inflammatory markers were not reviewed, the effect of treatment on cytokine storm could not be quantitatively measured.

\section{CONCLUSION}

In the current study, we did not find any statistically significant difference between those treated with montelukast as compared to placebo in any of the outcomes including progression to severe disease although only 1 person in the montelukast group needed ICU admission as compared to 3 in the placebo group.

\section{ACKNOWLEDGEMENTS}

Authors would like to acknowledge the COVID-19 management team consisting of the faculty from 
Department of General Medicine, Pulmonary Medicine, Anaesthesiology, Microbiology, Biochemistry, Haematology and Radiodiagnosis, who helped in setting up a dedicated COVID facility and their management protocol. Authors also thank all patients who participated in this study.

Funding: No funding sources

Conflict of interest: None declared

Ethical approval: The study was approved by the Institutional Ethics Committee

\section{REFERENCES}

1. Zhu N, Zhang D, Wang W, Li X, Yang B, Song J, et al. A Novel Coronavirus from Patients with Pneumonia in China, 2019. N Engl J Med. 2020;382(8):727-33.

2. Dexamethasone in Hospitalized Patients with Covid19. N Engl J Med. 2021;384(8):693-704.

3. Beigel JH, Tomashek KM, Dodd LE, Mehta AK, Zingman BS, Kalil AC, et al. Remdesivir for the Treatment of Covid-19 - Final Report. N Engl J Med. 2020;383(19):1813-26.

4. Repurposed Antiviral Drugs for Covid-19 - Interim WHO Solidarity Trial Results. N Engl J Med. 2021;384(6):497-511.

5. Salama C, Han J, Yau L, Reiss WG, Kramer B, Neidhart JD, et al. Tocilizumab in Patients Hospitalized with Covid-19 Pneumonia. N Engl J Med. 2021;384(1):20-30.

6. Group RC, Horby PW, Pessoa-Amorim G, Peto L, Brightling CE, Sarkar R, et al. Tocilizumab in patients admitted to hospital with COVID-19 (RECOVERY): preliminary results of a randomised, controlled, openlabel, platform trial. medRxiv. 2021.

7. Cadegiani FA. Repurposing existing drugs for COVID-19: an endocrinology perspective. BMC Endocr Disord. 2020;20(1):1-19.

8. López-Medina E, López P, Hurtado IC, Dávalos DM, Ramirez O, Martínez E, et al. Effect of Ivermectin on Time to Resolution of Symptoms Among Adults With Mild COVID-19: A Randomized Clinical Trial. JAMA. 2021;325(14):1426.

9. Echeverría-Esnal D, Martin-Ontiyuelo C, NavarreteRouco ME, De-Antonio Cuscó M, Ferrández O, Horcajada JP, Grau S. Azithromycin in the treatment of COVID-19: a review. Expert Rev Anti Infect Ther. 2021;19(2):147-63.

10. Fidan C, Aydoğdu A. As a potential treatment of COVID-19: Montelukast. Med Hypotheses. 2020;142:109828.

11. Lima-Morales R, Méndez-Hernández P, Flores YN, Osorno-Romero P, Sancho-Hernández CR, Cuecuecha-Rugerio E, et al. Effectiveness of a multidrug therapy consisting of Ivermectin, Azithromycin, Montelukast, and Acetylsalicylic acid to prevent hospitalization and death among ambulatory COVID-19 cases in Tlaxcala, Mexico. Int J Infect Dis. 2021;105:598-605.

12. Keeley P, Buchanan D, Carolan C, Pivodic L, Tavabie S, Noble S. Symptom burden and clinical profile of COVID-19 deaths: a rapid systematic review and evidence summary. BMJ Support Palliat Care. 2020;10(4):381-4.

13. Nile SH, Nile A, Qiu J, Li L, Jia X, Kai G. COVID19: Pathogenesis, cytokine storm and therapeutic potential of interferons. Cytokine Growth Factor Rev. 2020;53:66-70.

14. Chen X, Zhang X, Pan J. Effect of Montelukast on Bronchopulmonary Dysplasia (BPD) and Related Mechanisms. Med Sci Monit Int Med J Exp Clin Res. 2019;25:1886-93.

15. Wu AY, Chik SC, Chan AW, Li Z, Tsang KW, Li W. Anti-inflammatory effects of high-dose montelukast in an animal model of acute asthma. Clin Exp Allergy. 2003;33(3):359-66.

16. Noor A, Najmi MH, Bukhtiar S. Effect of Montelukast on bradykinin-induced contraction of isolated tracheal smooth muscle of guinea pig. Indian J Pharmacol. 2011;43(4):445-9.

17. Bozek A, Winterstein J. Montelukast's ability to fight COVID-19 infection. J Asthma. 2020;1-2.

18. Barré J, Sabatier JM, Annweiler C. Montelukast Drug May Improve COVID-19 Prognosis: A Review of Evidence. Front Pharmacol. 2020;11:1344.

19. Khan AR, Misdary C, Yegya-Raman N, Kim S, Narayanan N, Siddiqui $S$, et al. Montelukast in hospitalized patients diagnosed with COVID-19. J Asthma. 2021;0(0):1-7.

20. Tavares M, Farraia M, Silva S, Ribeiro AM, Severo M, Paciência I, et al. Impact of montelukast as add on treatment to the novel coronavirus pneumonia (COVID-19): protocol for an investigator-initiated open labeled randomized controlled pragmatic trial. Porto Biomed J. 2021;6(2).

21. Wilchesky M. Repurposing Montelukast for the Attenuation and Prophylaxis of Severe COVID-19 Symptoms: The COvid-19 Symptom MOntelukast (COSMO) Trial. Available at: https://clinical trials.gov/ct2/show/NCT04389411. Accessed on 25 April 2021.

22. Clinical Management Protocol for COVID-19. Available at: https://www.mohfw.gov.in/pdf/Clinical ManagementProtocolforCOVID19.pdf. Accessed on 29 April 2021.

Cite this article as: Kumar V, Ganapule AA, Supriya S, Bhushan D, Lohani P, Pandey S. Efficacy of montelukast in the management of COVID-19: double blind randomized placebo controlled trial. Int J Basic Clin Pharmacol 2021;10:1374-9. 